\title{
Recollection rejection: Gist cuing of verbatim memory
}

\author{
TIMOTHY N. ODEGARD \\ University of Texas, Arlington, Texas \\ and \\ JAMES M. LAMPINEN \\ University of Arkansas, Fayetteville, Arkansas
}

\begin{abstract}
Recollection rejection allows people to avoid accepting related lures presented on recognition tests by recollecting their instantiating study items. Two predictions made by fuzzy trace theorists were tested. First, recollection rejection should likely occur when verbatim traces are available for retrieval. Second, related lures that closely approximate the gist of their instantiating study items should be more likely to evoke recollection rejection than related lures that do not provide such a match. In support of the first condition, estimates of recollection rejection were lower when participants studied items under divided as opposed to full attention. In support of the second prediction, estimates of recollection rejection were greater when participants were presented with antonyms as related lures, as opposed to rhymes. The present results contribute to the cued recall and recognition literatures by demonstrating that recollection rejection is moderated by similar factors as is cued recall.
\end{abstract}

Historically, psychologists have investigated memory errors to explore the processes and representational characteristics of memory (see, e.g., Bartlett, 1932; Bransford \& Franks, 1972; Deese, 1959; Underwood, Kapelak, \& Malimi, 1976; Underwood \& Zimmerman, 1973). In recent years, interest has focused on understanding the processes by which false recognition occurs and the processes by which it is avoided (see, e.g., Brainerd, Reyna, \& Mojardin, 1999; Gallo, Roberts, \& Seamon, 1997; Hicks \& Marsh, 1999; Israel \& Schacter, 1997; Jacoby, 1991; 1996; McDermott \& Roediger, 1998; Neuschatz, Payne, Lampinen, \& Toglia, 2001; Roediger \& McDermott, 1995; Schacter, Israel, \& Racine, 1999). In the present research, we examine recollection rejection or recall to reject, ${ }^{1}$ an editing process that allows people to avoid falsely accepting related lures presented on recognition memory tests by recalling the lures' instantiating study items (Brainerd, Reyna, Wright, \& Mojardin, 2003; Gallo, 2004; Hintzman, Curran, \& Oppy, 1992; Lampinen, Odegard, \& Neuschatz, 2004; Rotello \& Heit, 1999; Rotello, Macmillan, \& Van Tassel, 2000; Tulving, 1983). For example, in past research, we presented participants with study items such as towtruck and tightrope, and later presented them with related lures such as towrope (Lampinen et al., 2004). Evidence from thought listing and confidence measures suggested that participants were often able to avoid falsely accepting these related lures, because they recalled the presentation

Correspondence concerning this article should be sent to T. N. Odegard, Department of Psychology, Box 19528, University of Texas, Arlington, TX 76019-0528 (e-mail: odegard@uta.edu). of the corresponding studied item (e.g., I know this word was not presented, because I saw towtruck, not towrope).

Several researchers have provided evidence for the role of recollection in the rejection of related lures and have appealed to several theoretical perspectives when explaining their data (see, e.g., Gallo, 2004; Hintzman et al., 1992). In this article, we address predictions about the occurrence of recollection rejection stipulated within fuzzy trace theory. Specifically, we address (1) the need for verbatim memory traces to be encoded for study items and (2) the characteristics of related lures needed to cue the recall of their corresponding studied items, allowing for the occurrence of recollection rejection.

According to fuzzy trace theorists, two independent memory traces, gist and verbatim, are encoded for every memorial event. A gist memory trace is a fuzzy representation of an event's underlying meaning and structural form. A verbatim memory trace is an exact representation of an event's surface level features. In regard to recognition memory, gist memory traces support similarity judgments for targets and related lures, and verbatim memory traces support recollection of targets and recollection rejection of related lures. Recollection rejection of a related lure is based on a nonidentity judgment that occurs when (1) a related lure evokes the retrieval of its corresponding studied item's verbatim memory trace, (2) the test item is compared to the memory trace, (3) a mismatch is observed, and (4) the test item is rejected.

Brainerd et al. (2003) argued that recollection rejection of related lures is most likely to occur when (1) participants have good memories for the surface level features of studied items (i.e., verbatim memory traces) and (2) re- 
lated lures serve as good cues for their corresponding studied items' verbatim memory traces. To the extent that recollection rejection is based on cued recall, Brainerd et al.'s findings about these processes are analogous to the interaction that occurs between the study and test environments to facilitate cued recall (see, e.g., Fisher \& Craik, 1977; Tulving, 1974, 1983), and to the availability/ accessibility distinction made by Tulving and Pearlstone (1966). Availability refers to the existence of a memorial representation for a previously experienced event. Accessibility refers to the ability of a person to gain access to a stored memory trace. In the first experiment, we divided our participants' attention at study in an attempt to limit their ability to store memory traces for studied items, limiting the number of verbatim memory traces that were accessible to them. In Experiments 2A and 2B, we manipulated the quality of the retrieval cue provided by related lures presented at test. We hypothesized that this manipulation should influence the ability of individuals to gain access to previously stored verbatim memory traces.

A person might think that cues that match the surface forms of the presented items would provide the best retrieval cues for verbatim traces. Indeed, in some of their writing, Brainerd and Reyna (see, e.g., Brainerd et al., 1999; Brainerd, Reyna, \& Kneer, 1995) have emphasized the role of surface level information shared between studied items and their corresponding related lures in facilitating recollection rejection. However, another line of research has stressed the importance of conceptual overlap shared between studied items and their corresponding related lures in facilitating recollection rejection (see Brainerd et al., 2003, for a review). Indeed, evidence indicates that overlapping meaning representations provide strong cues for the retrieval of surface level representations (Johnson-Laird, 1987). For example, the fish attacked the swimmer is better recalled when shark as opposed to fish is presented as a cue (Anderson \& Ortony, 1975). Because of the meaning of fish in the context of the sentence, shark captures the meaning or gist of the sentence better than fish. As a result, shark provides an excellent retrieval cue for the exact sentence that was previously presented. This is just one example of the gist cuing of a verbatim memory trace.

To address the accessibility of verbatim memory traces and the ability of related lures to serve as cues for accessing verbatim memory traces, we manipulated the level of attention with which participants viewed the study list in Experiment 1 and the relationship shared between study items and their corresponding related lures in Experiments 2A and 2B. Manipulating the level of attention with which participants studied a list of words should influence their ability to engage recollection rejection. In comparison with participants who study items under full attention, participants who study items under divided attention should have decreased rates of recollection rejection. In Experiments $2 \mathrm{~A}$ and 2B, we manipulated the relationship shared between related lures and their corresponding studied items. This was done in an attempt to understand the characteristics that related lures need to have in order to give rise to the recall of their instantiating studied items and recollection rejection. At the time of test, half of the participants were presented with related lures that rhymed with their corresponding studied items, and the other half were presented with related lures that meant the opposite of their corresponding studied items. This manipulation seemed particularly relevant to the investigation of recollection rejection, because related lures serve as extra study-list cues for the recall of their corresponding study items. There is a whole class of variables that moderate the ability of extralist cues to evoke the recall of studied items (see Nelson, 1989, for a review). Meaning-based associates are among these variables. Therefore, the manipulation introduced in Experiments 2A and 2B should moderate the occurrence of recollection rejection.

\section{EXPERIMENT 1}

In this experiment, we divided participants' attention at study in an attempt to decrease their ability to recollect what was presented to them at study (see, e.g., Debner \& Jacoby, 1994; Gardiner \& Parkin, 1990; Jacoby, 1996; Jacoby, Toth, \& Yonelinas, 1993). Decreasing participants' abilities to recollect what was presented at study should influence their ability to utilize related lures as cues for their instantiating studied items, allowing for recollection rejection to occur. In the context of fuzzy trace theory, differential rates of recollection rejection should be observed between the full and divided attention conditions, because participants who study items under full attention should be better able to encode verbatim memory traces for studied items than participants who study items under divided attention. Brainerd et al. (1999) theorized that manipulations such as dividing attention at encoding should have relatively little influence on the rate at which gist memory traces are encoded. Indeed, Wallace, Malone, and Spoo (2000; see also, Wallace, Stewart, Shaffer, \& Wilson, 1998) found the gist of an item to be processed preattentively. Therefore, dividing attention at encoding should differentially affect the encoding of gist and verbatim memory traces.

To obtain estimates of recollection rejection, we utilized conjoint recognition (see Brainerd et al., 1999). Like the process dissociation model (Jacoby, 1991, 1996), conjoint recognition is an opposition procedure that allows for the computation of theoretically implied memory processes. In conjoint recognition, a participant studies a single study list and later completes a recognition memory test containing three kinds of items (i.e., targets, related lures, and unrelated lures) when given one of three instruction sets (i.e., target, related, target + related). Target instructions request that participants accept only targets, test items that were presented at study. Related instructions request that participants accept only related lures, test items that were not presented at study but that are related 
to items that were presented at study. Target + related instructions request that participants accept both targets and related lures. Under all three instruction sets, participants are asked to reject unrelated lures.

A full treatment of the conjoint recognition model is provided by Brainerd et al. (1999). Because this article deals with recollection rejection, we describe how participants respond to related lures under the three different instruction sets. The acceptance of related lures under the three different instruction sets can be summarized in the following equations, where $p_{\mathrm{r}, \mathrm{T}}$ is the probability of accepting a related lure given target instructions, $p_{\mathrm{r}, \mathrm{R}}$ is the probability of accepting a related lure given related instructions, and $p_{\mathrm{r}, \mathrm{T}+\mathrm{R}}$ is the probability of accepting a related lure given target + related instructions.

$$
\begin{gathered}
p_{\mathrm{r}, \mathrm{T}}=\left(1-\mathrm{R}_{\mathrm{r}}\right) \mathrm{S}_{\mathrm{r}}+\left(1-\mathrm{R}_{\mathrm{r}}\right)\left(1-\mathrm{S}_{\mathrm{r}}\right) \mathrm{B}_{\mathrm{T}} \\
p_{\mathrm{r}, \mathrm{R}}=\mathrm{R}_{\mathrm{r}}+\left(1-\mathrm{R}_{\mathrm{r}}\right) \mathrm{S}_{\mathrm{r}}+\left(1-\mathrm{R}_{\mathrm{r}}\right)\left(1-\mathrm{S}_{\mathrm{r}}\right) \mathrm{B}_{\mathrm{R}} \\
p_{\mathrm{r}, \mathrm{T}+\mathrm{R}}=\mathrm{R}_{\mathrm{r}}+\left(1-\mathrm{R}_{\mathrm{r}}\right) \mathrm{S}_{\mathrm{r}} \\
\quad+\left(1-\mathrm{R}_{\mathrm{r}}\right)\left(1-\mathrm{S}_{\mathrm{r}}\right) \mathrm{B}_{\mathrm{T}+\mathrm{R}}
\end{gathered}
$$

Note that in Equation 1, recollection rejection $\left(\mathrm{R}_{\mathrm{r}}\right)$ results in the rejection of the related lure, but in Equations 2 and 3 , this same process results in the acceptance of the related lure. Similarity judgments (i.e., $\mathrm{S}_{\mathrm{r}}$ ) result in the acceptance of related lures under all three instruction sets. Opposition of this nature can be utilized to provide estimates for the occurrence of recollection rejection.

Crossing the three instruction sets with the three recognition item types produces an observed outcome space to which the 7-parameter conjoint recognition multinomial model can be fit using standard maximum-likelihood techniques (Brainerd et al., 1999; Riefer \& Batchelder, 1988). The 7-parameter conjoint recognition model is designed to provide estimates of four memory processes and estimates of response bias under each of the three instruction sets. For targets, the model provides estimates of recollection(i.e., verbatim) and similarity- (i.e., gist) based acceptances. For related lures, the model provides estimates of recollection-based rejections and similarity-based acceptances. Fitting the model in this fashion also allows for a goodness-of-fit estimator to be calculated testing the null hypothesis that the observed data fit the model. This is done by comparing the fit of a constrained 7-parameter model to that of a larger, unconstrained 9-parameter model. The goodness-of-fit estimator is asymptotically distributed as chi square, and the difference between the number of parameters being estimated in the constrained and unconstrained models provides the degrees of freedom needed for the log-likelihood ratio statistic (Riefer \& Batchelder, 1988).

\section{Method}

Design and Participants. Ninety college undergraduates participated in this study as partial fulfillment of a research requirement. The experiment conformed to a 2 (attention: full, divided) $\times$ 3 (instruction: target, related, target + related) factorial design. Half of the participants studied words under full attention, and the other half of the participants studied words under divided attention. In addition, participants completed the recognition memory test under one of three instructional sets.

Materials. A list of 60 familiar words and their antonyms were used as study and test materials. At study, participants viewed 40 words. All participants completed a 60 -item recognition memory test composed of 20 targets, 20 related lures, and 20 unrelated lures. Related lures were antonyms of words that were presented at study, and unrelated lures were not related to any of the words that were presented at study. The same 60 items were always presented at test. Materials were counterbalanced such that each word appeared equally often as a target, a related lure, and an unrelated lure.

Procedure. At study, participants viewed 40 words presented on a computer monitor at a rate of 1 word every $3 \mathrm{sec}$. The attention of the participants in the divided attention condition was divided at study by a number-monitoring task (Craik, 1982; Jacoby, 1991). For this task, participants monitored a list of numbers presented over a pair of headphones and indicated by pressing the space bar when they heard three odd numbers presented in a row. Digits were presented at $1-\mathrm{sec}$ intervals. Participants were explicitly instructed that tracking the numbers and attending to the study words were of equal importance. They were also informed that the computer was recording their performance on the number-monitoring task and that they would need to remember the words presented on the computer monitor in order to complete a memory test, which reiterated the importance of attending to both the words and the numbers.

Immediately following the presentation of the study words, participants were instructed on how to complete the recognition memory test. Participants received one of three types of instructions: accept targets, accept related lures, accept targets and related lures. The experimenter read the instructions aloud while participants followed along. Each of the three instructions began with the following information.

You saw a list of vocabulary words. Now, I'm going to give you a test to see what you can remember from the words that you saw. The test will proceed as follows. You will now see another list of vocabulary words. Some of the words will be old words that you saw earlier. The rest will be new words that you did not see earlier. Some of the new words will be completely UNRELATED to words that were presented earlier. But, other new words will be ANTONYMS of the words that were displayed to you on the computer monitor. An ANTONYM is a word that means the opposite of another word. For example, suppose you saw the word BIG earlier and you saw the word LITTLE on the test. LITTLE and BIG are antonyms. They mean the opposite. Likewise, suppose you saw the word STAND and you saw the word SIT on the test. STAND and SIT are antonyms. They mean the opposite.

You are now going to see the test list of vocabulary words. As you see each word, you should respond by pressing the keys on the keyboard labeled "YES" or "NO."

After this introductory description, the actual instructions for how participants should complete the recognition memory test were provided. For the target instructions, participants were asked in the following manner to accept only targets.

Press "YES" whenever you see an old word that was presented earlier. Press "NO" whenever you see a new word that was not presented earlier. Please do not guess. Only press "YEs" when you remember having seen a word. Be very careful not to press "YES" when you see new words that are antonyms of words that you did see earlier.

For the related instructions, participants were asked in the following manner to accept only related lures.

Press "YES" whenever you see an antonym of an old word that was presented earlier. Press "NO" whenever you see an old word that was presented earlier, and press "NO" whenever you see a new word that is not an antonym of an old word. So, press "YES" whenever you see an antonym of an old word, but press "NO" for all other words. Please do not guess. Only press "YEs" when a word is an antonym of a word that you saw earlier. 
For the target + related instructions, participants were asked to accept targets and related lures.

Press "YES" whenever you see an old word that was presented earlier. Also, press "YEs" whenever you see a new word that is an antonym of an old word. Press "NO" for all other new words that are not antonyms of old words. So, press "YES" whenever you see an old word, press "YES" whenever you see an antonym of an old word, and press "NO" for all other words. Please do not guess. Only press "YEs" when a word is one you remember having seen earlier or when it is an antonym of a word presented earlier.

After the experimenter answered any questions asked by participants, participants were required to complete a practice test to demonstrate their comprehension of the instructions. For the practice test, participants were asked to suppose that on the study vocabulary list they had seen the words SOLDIER, PIG, STAND, COP, NEW. They were then asked to indicate how they would respond to SOLDIER, ROBBER, POP, NEW, BAND if they were on a test. Participants had to pass this test before they were allowed to begin the recognition test. Participants then completed a 60 -item recognition memory test. Each recognition test item was presented in the center of the computer screen. Participants responded "yes" or "no" by pressing corresponding keys on a keyboard. Responding to a test item caused the next test item to be presented.

\section{Results and Discussion}

The rates of responding "yes" to the different types of recognition items under the three different instruction sets are presented in Table 1, with standard deviations provided in parentheses. In addition, these data were fit to the 7-parameter conjoint recognition model. This was achieved by calculating the frequency at which all of the participants accepted the different item types under the three different instruction sets and fitting these values to the model using Hu's (1995) General Processing Tree program. A significance level of $p<.05$ was used for all analyses reported below, which corresponds to critical chi-square values of 3.84 for all analyses with $1 d f$ and 5.99 for all analyses with $2 d f$. The parameter estimates obtained from fitting these data are presented in Table 2, with standard deviations provided in parentheses. As is apparent from the chi-square values provided in Table 2, the model provided excellent fits of both data sets.
Of the memory estimates provided by the model, we were most interested in recollection and recollection rejection. We expected to replicate the typical finding that dividing attention at study decreases participants' ability to recollect the past. In addition, we hypothesized that if recollection rejection is indeed dependent on the accessibility of verbatim traces, then dividing attention at study should reduce the ability of participants to engage recollection at the time of test to oppose the false acceptance of related lures. Dividing attention at study significantly decreased the parameter estimate of recollection of targets $\left[\chi^{2}(1)=10.72\right]$. Furthermore, dividing attention at study significantly decreased the parameter estimate of recollection rejection $\left[\chi^{2}(1)=6.47\right]$. Dividing attention did not significantly decrease parameter estimates of similarity for related lures $\left[\chi^{2}(1)=.01\right]$. Participants did accept more targets based on similarity judgments when attention was not divided at study, but this difference failed to reach statistical significance $\left[\chi^{2}(1)=2.09\right]$. Dividing attention decreased the ability of participants to engage recollection while leaving unaffected the ability of participants to engage similarity. This was true both for the recollection-based acceptance of targets as well as the recollection-based rejection of related lures.

\section{EXPERIMENTS 2A AND 2B}

When discussing the prediction that recollection rejection should occur when related lures match the gist of studied items, a distinction needs to be made between the characteristics of a related lure and the characteristics of the information retrieved. As the early fish/shark example shows, a gist-based retrieval cue may cue the recall of a verbatim memory trace for exactly what was presented earlier. In Experiments 2A and 2B, we manipulated the type of relationship shared between related lures and their corresponding studied items.

In both experiments, half of the participants were presented with antonyms as related lures, and the remaining

Table 1

Mean Proportions of Items Accepted (With Standard Deviations) as Old in Experiments 1, 2A, and 2B

\begin{tabular}{|c|c|c|c|c|c|c|c|c|c|c|c|c|}
\hline & \multicolumn{4}{|c|}{ Experiment 1} & \multicolumn{4}{|c|}{ Experiment $2 \mathrm{~A}$} & \multicolumn{4}{|c|}{ Experiment 2B } \\
\hline & \multicolumn{2}{|c|}{ Full } & \multicolumn{2}{|c|}{ Divided } & \multicolumn{2}{|c|}{ Antonyms } & \multicolumn{2}{|c|}{ Rhymes } & \multicolumn{2}{|c|}{ Antonyms } & \multicolumn{2}{|c|}{ Rhymes } \\
\hline & $M$ & $\overline{S D}$ & $M$ & $\overline{S D}$ & $M$ & $\overline{S D}$ & $M$ & $\overline{S D}$ & $M$ & $\overline{S D}$ & $M$ & $\overline{S D}$ \\
\hline \multicolumn{13}{|l|}{ Targets } \\
\hline $\mathrm{T}$ instructions & .76 & .14 & .58 & .17 & .68 & .15 & .73 & .14 & .70 & .15 & .66 & .22 \\
\hline $\mathrm{R}$ instructions & .18 & .27 & .29 & .14 & .21 & .22 & .23 & .17 & & & & \\
\hline $\mathrm{T}+\mathrm{R}$ instructions & .78 & .14 & .67 & .15 & .65 & .12 & .73 & .16 & & & & \\
\hline \multicolumn{13}{|l|}{ Related Lures } \\
\hline $\mathrm{T}$ instructions & .09 & .06 & .28 & .18 & .19 & .16 & .22 & .16 & .27 & .17 & .24 & .19 \\
\hline $\mathrm{R}$ instructions & .58 & .12 & .46 & .16 & .50 & .19 & .34 & .13 & & & & \\
\hline $\mathrm{T}+\mathrm{R}$ instructions & .60 & .13 & .55 & .15 & .58 & .14 & .46 & .16 & & & & \\
\hline \multicolumn{13}{|l|}{ Unrelated Lures } \\
\hline $\mathrm{T}$ instructions & .06 & .07 & .29 & .22 & .17 & .14 & .17 & .15 & .21 & .16 & .18 & .13 \\
\hline $\mathrm{R}$ instructions & .15 & .16 & .29 & .18 & .29 & .16 & .29 & .23 & & & & \\
\hline $\mathrm{T}+\mathrm{R}$ instructions & .26 & .15 & .38 & .16 & .38 & .14 & .26 & .13 & & & & \\
\hline
\end{tabular}


Table 2

Parameter Estimates From Experiments 1 and 2A

\begin{tabular}{|c|c|c|c|c|c|c|c|c|}
\hline & \multicolumn{4}{|c|}{ Experiment 1} & \multicolumn{4}{|c|}{ Experiment 2A } \\
\hline & \multicolumn{2}{|c|}{ Full } & \multicolumn{2}{|c|}{ Divided } & \multicolumn{2}{|c|}{ Antonyms } & \multicolumn{2}{|c|}{ Rhymes } \\
\hline & $M$ & $S D$ & $M$ & $S D$ & $M$ & $S D$ & $M$ & $\mathrm{SD}$ \\
\hline Recollection (targets) & .59 & .03 & .30 & .04 & .53 & .03 & .54 & .04 \\
\hline Recollection rejection & .43 & .03 & .18 & .04 & .26 & .04 & .14 & .04 \\
\hline Similarity (targets) & .33 & .04 & .19 & .04 & .11 & .05 & .25 & .05 \\
\hline Similarity (related) & .09 & .03 & .08 & .04 & .12 & .04 & .12 & .03 \\
\hline \multicolumn{9}{|l|}{ Bias Parameters } \\
\hline Target instructions & .07 & .01 & .28 & .03 & .15 & .02 & .15 & .02 \\
\hline Related instructions & .15 & .02 & .29 & .02 & .23 & .03 & .16 & .02 \\
\hline Target + Related instructions & .25 & .02 & .40 & .02 & .29 & .03 & .25 & .02 \\
\hline$\chi^{2}(2)$ & \multicolumn{2}{|c|}{1.44} & \multicolumn{2}{|c|}{70} & \multicolumn{2}{|c|}{3.90} & \multicolumn{2}{|c|}{2.56} \\
\hline
\end{tabular}

participants were presented with rhymes as related lures. In his direct comparison experiment, Tulving (1983) provided evidence that strong associates (e.g., antonyms) provided better retrieval cues, resulting in higher rates of recollection rejection, than did rhymes. Other research from the cued recall literature would suggest that meaningbased relationships make relatively better recall cues than perceptually based recall cues (Fisher \& Craik, 1977; Nelson, Walling, \& McEvoy, 1979). Given this past research, we too expected antonyms to provide better cues, resulting in greater levels of recollection rejection than with rhymes.

In Experiment 2A, the conjoint recognition model was again used to provide parameter estimates of recollection rejection. In Experiment 2B, a thought-listing paradigm was used, in which participants wrote an explanation for why they accepted or rejected each of the recognition test items. If recollection rejection results from the retrieval of a verbatim memory trace, participants should be able to report this occurrence. When participants reported rejecting a related lure because they recalled the presentation of its antonym or rhyme at study, the related lure was considered to have been rejected based on recollection rejection. A stringent criterion was used when identifying recollection rejection in this manner. Participants had to recall and report the exact item that was presented when rejecting a related lure (e.g., it was not dog, it was cat). In this manner, we obtained self-report-based estimates for the occurrence of recollection rejection. These data were used to provide converging evidence for the general pattern of findings in Experiment 2A. These data also provided a description of the phenomenology that accompanies the rejection of some recognition test items.

\footnotetext{
Method

Design and Participants. One hundred eight college undergraduates participated in Experiment 2A as partial fulfillment of a research requirement. The experiment conformed to a 2 (relation: antonym, rhyme) $\times 3$ (instruction: target, related, target + related) factorial design. The relationship shared between studied items and their corresponding related lures was manipulated between participants. Participants were presented with either antonyms or rhymes as related lures at test. In addition, participants completed a recognition memory test under one of three conjoint recognition instructional sets.
}

Thirty-six participants participated in Experiment 2B. In this experiment, participants were always presented with target instructions. As in Experiment 2A, the relationship of the related lure to its instantiating study item was manipulated between participants. In addition, participants in this experiment were required to provide explanations for why they accepted or rejected each of the recognition test items after first providing "yes/no" responses to all of the test items.

Materials. A list of 45 familiar words and their antonyms and rhymes was used as study and test material. At study, 30 words were presented. All participants completed a 45 -item recognition memory test composed of 15 targets, 15 related lures, and 15 unrelated lures. Fewer items were included in the study and test phases of this experiment because of constraints on the rhyming associates.

Rhyming associates were obtained by having 75 individuals report the first rhyming word that came to mind when they were presented with the 120 words that were used to construct the materials for the first experiment. In a pair of antonyms, a word was selected to be the critical item in the pair when it had the stronger of the two rhyming associates. By critical item, we mean that this was the item that would be included on the recognition test as a target, a related lure, or an unrelated lure. There were two exceptions to this general rule. In two cases, the largest rhyming associate was semantically related to the word (flood, mud and night, light). In these cases, the other word in an antonym pair (i.e., drought, day) was used as the target word and was presented at test.

Procedure. The procedure for this experiment was almost identical to that of the full attention condition of the first experiment, with one exception. Participants listened to a list of words that were presented over a pair of headphones. This change was made to help ensure that participants were exposed to the phonology of the studied items. As in the first experiment, words were presented at a rate of approximately one word every $3 \mathrm{sec}$. In addition, each recognition item was played over a pair of headphones, and participants responded "yes" on "no" before advancing to the next item. For Experiment $2 \mathrm{~A}$, the conjoint recognition instructions were modified for the change in modality, and an additional set of instructions was constructed for the rhyme condition. All participants in the rhyme condition were first presented with and read a general description.

You heard a list of vocabulary words earlier. Now, I'm going to give you a test to see what you can remember from the words that you heard. The test will proceed as follows. You will now hear another list of vocabulary words. Some of the words will be old words that you heard earlier. The rest will be new words that you did not hear earlier. Some of the new words will be completely UNRELATED to words that you heard But, other new words will be RHYMES of the words that you heard. A RHYMING word sounds similar to another word. For example, suppose you heard the word BIG earlier and you hear the word PIG on the test list. PIG and BIG are RHYMES. They sound similar. Likewise, suppose you heard the word STAND earlier and you hear the word BAND on the test list. STAND and BAND are RHYMES. They sound similar. You are now 
going to hear the test list of vocabulary words. As you hear each word, you should respond by pressing the keys on the keyboard labeled "YES" or "NO."

Those participants receiving target instructions were then asked to accept only targets.

Press "YES" whenever you hear an old word that was played over the headphones earlier. Press "NO" whenever you hear a new word that was not presented earlier. Please do not guess. Only press "YEs" when you remember having heard a word before. Be very careful not to press "YES" when you hear new words that are RHYMES of words that you heard earlier.

Those participants receiving related instructions were then asked to accept only related lures.

Press "YES" whenever you hear a RHYME of an old word that was played over the headphones earlier. Press "NO" whenever you hear an old word that was presented earlier, and press "NO" whenever you hear a new word that is not a RHYME of an old word. So, press "YES" whenever you hear a RHYME of an old word, but press "NO" for all other words. Please do not guess. Only press "YES" when a word RHYMES with a word that you heard earlier.

Those participants receiving target + related instructions were then asked to accept only targets and related lures.

Press "YES" whenever you hear an old word that was played over the headphones earlier. Also, press "YEs" whenever you hear a new word that is a RHYME of an old word. Press "NO" for all other new words that are not RHYMES of old words. So, press "YES" whenever you hear an old word, press "YES" whenever you hear a RHYME of an old word, and press "NO" for all other words. Please do not guess. Only press "YES" when a word is one you remember having heard earlier or when it RHYMES with a word that you heard earlier.

Participants in Experiment 2B were provided with target instructions. After completing the recognition memory test, these participants were asked to provide a written explanation for why they accepted or rejected each of the items.

\section{Results: Experiment 2A}

Rates of accepting the recognition test items under the three different instruction sets are presented in Table 1. As before, data obtained in this experiment were fit to the 7-parameter conjoint recognition model, and parameter estimates are provided in Table 2. As is apparent from the chi-square values in Table 2, the model provided excellent fits of both data sets.

Again we were most interested in the recollection parameters of the model, predicting that our participants' abilities to recollect targets would be identical between the rhyme and antonym conditions. As can be seen in Table 2, this was indeed the case $\left[\chi^{2}(1)=0.27\right]$. The prediction that participants presented with antonyms as related lures would be better able to engage recollection rejection than participants presented with rhymes as related lures was confirmed as well $\left[\chi^{2}(1)=4.5\right] .^{2}$ There were no significant differences in the rate at which participants accepted related lures based on similarity judgments between the two conditions $\left[\chi^{2}(1)=.01\right]$. Participants in the rhyme condition accepted more targets based on similarity than participants in the antonym condition, but this difference failed to reach statistical significance $\left[\chi^{2}(1)=3.25\right]$.

\section{Results: Experiment 2B}

Behavioral data obtained in this experiment are reported in Table 1. The data of interest to our present discussion are the self-reported reasons why participants accepted or rejected the test items. Two individuals coded the explanations given by participants for why they rejected a test item for instances of various memorial processes. Recollection rejection was among these rejection processes. For a test item to have been given a rating of recollection rejection, a participant had to report rejecting an item because he/she recalled the presentation of another item. When this occurred, raters coded for whether or not the recalled word was actually presented at study. As a result, participants could report the phenomenology of recollection rejection for targets, related lures, and unrelated lures. But only when participants were presented with a related lure could the recalled word actually have been presented at study. On average, participants reported the phenomenology of recollection rejection less than .02 of the times for targets and unrelated lures.

To determine whether participants varied in their rates of self-reported recollection rejection when presented with antonyms as opposed to rhymes as related lures, we conducted an independent samples $t$ test on the proportion of related lures rejected using recollection rejection in the two conditions. Participants reported using recollection rejection significantly more often when presented with related lures that were antonyms $(M=.16, S D=$ $.18)$ as opposed to rhymes $(M=.05, S D=.06)[t(34)=$ 2.43]. Qualitatively, these data replicate the data obtained in Experiment 2A.

Participants' explanations for rejecting test items were also coded for rejection processes other than recollection rejection. Specifically, responses were coded for instances of nonspecific recollection rejection, distinctiveness rejection, and familiarity-based rejection. Nonspecific recollection rejection corresponded to a participant reportedly rejecting a test item based on the memory of a word that was presented at study, but failing to report exactly which word was presented at study. Distinctiveness rejections are instances of participants rejecting an item because, they said, if it had been presented, they would have remembered it having been presented. This classification was based on the distinctiveness heuristic outlined by Schacter and his colleagues (Israel \& Schacter, 1997; Schacter et al., 1999). Familiarity-based rejections correspond to participants reportedly rejecting an item because it seemed unfamiliar. Of these processes, participants self-reported rejecting test items based on familiarity, but did not self-report using the other processes. Moreover, participants self-reported familiarity-based rejections more often for rhyming lures $(M=.66, S D=.21)$ than for antonym lures $(M=.46, S D=.25)[t(34)=2.59]$.

\section{Discussion}

We observed that antonyms evoked greater rates of recollection rejection than rhymes in both Experiments $2 \mathrm{~A}$ and $2 \mathrm{~B}$. Conjoint recognition provided estimates of recollection rejection based on the rate at which participants rejected related lures under the target instructions and accepted these items under the related and target + related instructions. When given related instructions, participants 
were better able to accept related lures that were antonyms of studied items than related lures that were rhymes of studied items. Participants' increased ability to control behavioral responding when presented with antonyms, which was observed using conjoint recognition, was linked with the phenomenology of recollection. When asked to explain why they had rejected related lures, participants reported doing so because they recalled the instantiating study item more often when presented with antonyms than when presented with rhymes.

\section{GENERAL DISCUSSION}

There is growing interest in identifying mechanisms that allow individuals to successfully edit their recognition memory performance. The research presented in this article was intended to investigate two factors that have been suggested to facilitate the occurrence of one such editing process, recollection rejection. In the context of fuzzy trace theory, recollection occurs when participants have access to verbatim memory traces that represent the surface level features of studied items. The accessibility of verbatim memory traces should influence the ability of participants to recollect actually studied items and to use this information when accepting these items and when rejecting related items. In accordance with this hypothesis, we observed that dividing attention at study decreases estimates of both recollection of targets and recollection rejection of related lures provided by conjoint recognition.

Past researchers have observed that dividing attention at study decreases estimates of recollection of targets (see, e.g., Debner \& Jacoby, 1994; Gardiner \& Parkin, 1990; Jacoby, 1996; Jacoby et al., 1993). These data replicate and extend these previous findings to the domain of recollection rejection and semantically related distractors. Dividing attention decreased estimates of recollection for targets, but it also decreased estimates of recollection rejection of related lures. Decreasing the accessibility of verbatim traces to participants limited participants' ability to retrieve an item's verbatim memory trace when they were presented with the item as a cue (i.e., target) and its antonym as a cue (i.e., related lure). Both recollection and recollection rejection responded in predictable ways when attention was divided at encoding.

Our predictions about the effect of dividing attention at study were based on precepts of fuzzy trace theory. However, other theoretical perspectives make similar predictions. For example, Hintzman et al. (1992) appealed to MINERVA2 when accounting for the role of recall in the rejection of related lures. Dividing attention at study should decrease the overall memory strength of traces stored in long-term memory and the ability of participants to recall these traces when cued with their corresponding related lures. Jones and Jacoby (2001) made similar predictions about the effects of dividing attention on recollection rejection by appealing to Jacoby's (1991) recollection-familiarity distinction. They too predicted that dividing attention at study would decrease recollection and recollection rejection.

In addition, Jones and Jacoby (2001) suggested that the characteristics of the related lures should influence rates of recollection rejection. The related lures used in their research shared considerable perceptual overlap with their corresponding study items but minimal conceptual overlap with their corresponding items. Jones and Jacoby hypothesized that increasing the conceptual overlap shared between a related lure and its instantiating study item would facilitate the ability of the lure to serve as an effective retrieval cue. In support of this prediction, we were able to manipulate characteristics of the related lures themselves and moderate estimates of recollection rejection while leaving estimates of recollection unaffected. To do so, we manipulated the type of gist similarity shared between a study item and its corresponding related lure. We observed that related lures that shared a meaning-based association with their corresponding studied items were rejected based on recollection more often than were related lures that shared a perceptually based association with their corresponding studied items. Parameter estimates of recollection rejection provided by conjoint recognition were greater in the antonym condition than in the rhyme condition. Participants also self-reported exactly which word was presented at study more often when rejecting antonyms as opposed to rhymes, providing converging evidence for the role of gist in cuing verbatim memory traces.

Furthermore, the data obtained in the present experiments converge with past findings from the cued recall literature. Variables such as category set size, association strength, and the type of retrieval cue (e.g., category name, meaningful associate, rhyme) influence the ability of extralist words to cue the recall of studied items. Related lures are in essence extralist cues, and the findings in our research would suggest that recollection rejection (i.e., recall to reject) is moderated by similar factors. One set of factors that was not manipulated in Experiments $2 \mathrm{~A}$ and $2 \mathrm{~B}$ that potentially could have increased the rate of recollection rejection for related lures was the characteristics of the studied items processed at encoding. Past research has demonstrated that transfer-appropriate processing facilitates recall. If we had provided orienting tasks focusing participants on how the studied items sound, it might have increased estimates of recollection rejection for rhyming lures. However, Fisher and Craik (1977) concluded, based on their research, that matching the characteristics of information attended to at study and the characteristics of cues presented at test has relatively weak facilitating effects when rhymes are used as cues.

When seen from other theoretical perspectives, such as MINERVA2, antonyms may have allowed for greater rates of recollection because of the density of the search space. Each word has a considerable number of rhymes but only a limited number of antonyms. Restricting the search space in this manner should facilitate the recall 
process and recollection rejection (see also Norman \& O'Reilly, 2003). In the context of fuzzy trace theory, reducing the overall category size should make it easier for participants to identify a contrast between retrieved traces and the test item.

One might wonder whether participants engaged in recollection rejection because the conjoint recognition instructions requested them to do so. (The instructions used in these experiments explicitly informed participants of the relationships shared between related lures and their corresponding studied items.) However, the present data do not seem to warrant this conclusion. The instructions informed participants of the relationships shared between related lures in both the rhyme and the antonym conditions. However, participants rarely engaged in recollection rejection when presented with rhyming lures. This was the case even though the rhymes were normalized to ensure that related lures were the strongest rhyming associates of the targets. Moreover, we have observed recollection rejection even when participants were not provided with such detailed information about the study and test contexts (Lampinen et al., 2004; however, see Gallo, 2004).

It should also be pointed out that the recognition data provided in Table 1 do not provide direct evidence that the independent variables introduced in these three experiments had an impact on recollection rejection. Strictly speaking, the recognition data do not suggest that participants necessarily relied on their ability to recall an instantiating studied item when making a recognition judgment, but it should be pointed out that old/new recognition data can never conclusively demonstrate that participants rely on recall. As a result, like others, we began employing alternate routes to tap recollection rejection. What the present data demonstrate is that people can access additional information at a conscious level of awareness when presented with certain types of related lures, and that this information can be employed to enhance their recognition performance. Moreover, we strongly believe it is important to know that recollection rejection can be tapped, and what conditions facilitate its occurrence.

Across these experiments, the accessibility of verbatim memory influenced estimates of recollection and recollection rejection. We also observed that the quality of the related lure to serve as a retrieval cue for its instantiating item moderated recollection rejection. This article began with an appeal to the past and the historical use of memory errors. Collectively, the extant research has allowed psychologists to understand how recognition and recall function. Moreover, recall is known to play a role in recognition memory performance. In line with past research that has investigated recollection rejection/recall to reject, the research presented here highlights the role that cued recall can play in facilitating accurate memory performance.

In line with fuzzy trace theory, we have posited that meaning matters in recognition memory. As with the results obtained by Mandler (1980), our data point to the role of meaning in recollection or retrieval-based processes. Of course, from a fuzzy trace theory perspective, gist cues the retrieval of an independent, bound verbatim memory trace representing the surface features of the previous study item. Future research could address whether or not other representational characteristics of the study and test materials used in these experiments could also account for these results. In particular, future researchers might attempt to identify whether associations and category size, which influence cued recall, also influence the occurrence of recollection rejection.

To conclude, the ability to control the level of consciousness with which a person completes a recognition memory test (e.g., a multiple choice test or a photo lineup) has important implications for educational and forensic settings. An eyewitness who is able to describe, in detail, the culprit who actually committed a crime when faced with a lineup of innocent people, is extremely helpful. A person merely stating that none of the people in the lineup look familiar has the same immediate outcome - an innocent person is not unduly incriminated-but the more detailed information provided through recollection rejection is more likely to push the case forward. A student who explicitly states the truthful counterpart of a false statement on a true/false question has an additional learning experience. Moreover, doing so may "inoculate" the student against the contaminating effects of the inaccurate information contained in such educational situations. Even if employing different forms of related lures would unfortunately not influence the overall recognition data (i.e., false alarm rates), there are real life applications that would benefit by inducing recollection rejection.

\section{REFERENCES}

Anderson, R. C., \& Ortony, A. (1975). On putting apples into bottles - a problem of polysemy. Cognitive Psychology, 7, 167-180.

Bartlett, F. C. (1932). Remembering: A study in experimental and social psychology. Cambridge: Cambridge University Press.

Brainerd, C. J., Reyna, V. F., \& KneER, R. (1995). False-recognition reversal: When similarity is distinctive. Journal of Memory \& Language, 34, 157-185.

Brainerd, C. J., Reyna, V. F., \& Mojardin, A. H. (1999). Conjoint recognition. Psychological Review, 106, 160-179.

Brainerd, C. J., Reyna, V. F., Wright, R., \& Mojardin, A. H. (2003). Recollection rejection: False-memory editing in children and adults. Psychological Review, 110, 762-784.

Bransford, J. D., \& Franks, J. J. (1972). The abstraction of linguistic ideas: A review. Cognition, 1, 211-249.

Craik, F. I. M. (1982). Selective changes in encoding as a function of reduced processing capacity. In F. Klix, J. Hoffman, \& E. van der Meer (Eds.), Cognitive research in psychology (pp. 152-161). Berlin: Deutscher Verlag der Wissenschaften.

DeBner, J. A., \& JACOBY, L. L (1994). Unconscious perception: Attention, awareness, and control. Journal of Experimental Psychology: Learning, Memory, \& Cognition, 20, 304-317.

DEESE, J. (1959). On the prediction of occurrence of particular verbal intrusions in immediate recall. Journal of Experimental Psychology, 58, 17-22.

Fisher, R. P., \& CRAIK, F. I. M. (1977). Interaction between encoding and retrieval operations in cued recall. Journal of Experimental Psychology: Human Learning \& Memory, 3, 701-711. 
GALlo, D. A. (2004). Using recall to reduce false recognition: Diagnostic and disqualifying monitoring. Journal of Experimental Psychology: Learning, Memory, \& Cognition, 30, 120-128.

Gallo, D. A., Roberts, M. J., \& Seamon, J. G. (1997). Remembering words not presented in lists: Can we avoid creating false memories? Psychonomic Bulletin \& Review, 4, 271-276.

GARDInER, J. M., \& PARKIN, A. J. (1990). Attention and recollective experience in recognition memory. Memory \& Cognition, 18, 579-583.

HiCKS, J. L., \& MARSH, R. L. (1999). Attempts to reduce the incidence of false recall with source monitoring. Journal of Experimental Psychology: Learning, Memory, \& Cognition, 25, 1195-1209.

Hintzman, D. L., Curran, T., \& Oppy, B. (1992). Effects of similarity and repetition on memory: Registration without learning? Journal of Experimental Psychology: Learning, Memory, \& Cognition, 18, 667-680.

Hu, X. (1995). General Processing Tree (Version 1.1) [Computer software]. Irvine, CA.: University of California.

IsRAeL, L., \& SchACTER, D. L. (1997). Pictorial encoding reduces false recognition of semantic associates. Psychonomic Bulletin \& Review, 4, 577-581.

JACOBY, L. L. (1991). A process dissociation framework: Separating automatic from intentional uses of memory. Journal of Memory \& Language, 30, 497-514.

JACOBY, L. L. (1996). Dissociating automatic and consciously controlled effects of study/test compatibility. Journal of Memory \& Language, 35, 32-52.

Jacoby, L. L., Toth, J. P., \& Yonelinas, A. P. (1993). Separating conscious and unconscious influences of memory. Journal of Experimental Psychology: General, 122, 139-154.

JOHNSON-LAIRD, P. N. (1987). The mental representation of the meaning of words. Cognition, 25, 189-211.

Jones, T. C., \& JACOBY, L. L. (2001). Feature and conjunction errors in recognition memory: Evidence for dual process theory. Journal of Memory \& Language, 45, 82-102.

Lampinen, J. M., Odegard, T. N., \& Neuschatz, J. S. (2004). Robust recollection rejection in the memory conjunction paradigm. Journal of Experimental Psychology: Memory, Learning \& Cognition, 30, 332-342.

MANDLER, G. (1980). Recognizing: The judgment of previous occurrence. Psychological Review, 87, 252-271.

McDermott, K. B., \& Roediger, H. L., III (1998). Attempting to avoid illusory memories: Robust false recognition of associates persists under conditions of explicit warnings and immediate testing. Journal of Memory \& Language, 39, 508-520.

NELSON, D. L. (1989). Implicitly activated knowledge and memory. In C. Izawa (Ed.), Current issues in cognitive process: The Tulane Floweree Symposium on cognition (pp. 367-387). Hillsdale, NJ: Erlbaum.

Nelson, D. L., Walling, J. R., \& McEvoy, C. L. (1979). Doubts about depth. Journal of Experimental Psychology: Learning, Memory, \& Cognition, 5, 24-44.

Neuschatz, J. S., Payne, D. G., Lampinen, J. M., \& Toglia, M. P. (2001). Assessing the effectiveness of warnings and the phenomenological characteristics of false memories. Memory, 9, 39-51.

Norman, K. A., \& O’Reilly, R. C. (2003). Modeling hippocampal and neocortical contributions to recognition memory: A complementary learning systems approach. Psychological Review, 110, 611-646.

Riefer, D. M., \& BAtchelder, W. H. (1988). Multinomial modeling and the measurement of cognitive processes. Psychological Review, 95, 318-339.

Roediger, H. L., \& McDermott, K. B. (1995). Creating false memo- ries: Remembering words not presented in lists. Journal of Experimental Psychology: Learning, Memory, \& Cognition, 21, 803-814.

Rotello, C. M., \& Heit, E. (1999). Two process models of recognition memory: Evidence for recall-to-reject? Journal of Memory \& Language, 40, 432-453.

Rotello, C. M., Macmillan, N. A., \& Van Tassel, G. (2000). Recall-toreject in recognition: Evidence from ROC curves. Journal of Memory \& Language, 43, 67-88.

Schacter, D. L., Israel, L., \& Racine, C. (1999). Suppressing false recognition in younger and older adults: The distinctiveness heuristic. Journal of Memory \& Language, 40, 1-24.

Tulving, E. (1974). Cue dependent forgetting. American Scientist, 62 74-82.

Tulving, E. (1983). Elements of episodic memory. New York: Oxford University Press.

Tulving, E., \& Pearlstone, Z. (1966). Availability versus accessibility of information in memory for words. Journal of Verbal Learning \& Verbal Behavior, 5, 381-391.

Underwood, B. J., KaPelaK, S. M., \& Malimi, R. A. (1976). Integration of discrete verbal units in recognition memory. Journal of Experimental Psychology: Human Learning \& Memory, 2, 293-300.

Underwood, B. J, \& Zimmerman, J. (1973). The syllable as a source of error in multisyllable word recognition. Journal of Verbal Learning \& Verbal Behavior, 12, 701-706.

Wallace, W. P., Malone, C. P., \& Spoo, A. D. (2000). Implicit word activation during prerecognition processing: False recognition and remember/know judgments. Psychonomic Bulletin \& Review, 7, 149157.

Wallace, W. P., Stewart, M. T., Shaffer, T. R, \& Wilson, J. A. (1998). Are false recognitions influenced by prerecognition processing? Journal of Experimental Psychology: Learning, Memory, \& Cognition, 24, 299-315.

\section{NOTES}

1. Recollection rejection and recall to reject are two terms in the literature that have been introduced to describe the ability to reject related lures based on the recollection/recall of their instantiating studied items. In the context of this article, we adopt the term recollection rejection. We do so because we address fundamental predictions drawn from fuzzy trace theory that apply to this process. However, the recall to reject literature bears directly on the research that we conducted, and therefore, we cite this literature often.

2. Careful investigation of the acceptance rates provided in Table 1 make it quite obvious that recollection rejection is being mediated by the ability of a related lure to cue the recall of its instantiating studied item when participants are given related instructions. This is in contrast to the rate at which these items are accepted under $\mathrm{T}$ and $\mathrm{T}+\mathrm{R}$ instructions. As such, in Experiment 2A, what the recollection rejection parameter is measuring is the increased ability of antonyms to cue the recall of their instantiating studied items in comparison to rhymes. This result has been found in cued recall paradigms. What the present results do is extend these previous cued recall results into the domain of how recall is utilized in recognition memory, allowing participants to reject related lures. Although we have known what makes a good recall cue, a systematic application of this literature to that of recollection rejection/recall to reject has yet to be done.

(Manuscript received March 10, 2004; revision accepted for publication December 29, 2004.) 